\title{
Effects of Quantization on Golay Sequence based Channel Estimation
}

\author{
Márton Kovács, Barna Csuka, Zsolt Kollár \\ Budapest University of Technology and Economics \\ Budapest, Hungary \\ \{csuka,kollar\}@hvt.bme.hu
}

\begin{abstract}
In this paper we investigate a widely adapted complementary Golay sequences which are used for channel estimation in digital data transmission systems. The performance of the Golay correlator based and the fast Fourier transform based channel estimation methods are evaluated. The effects of quantization on the estimation of the channel impulse response is compared through simulations.

Index Terms-Golay correlator, Discrete Fourier Transform, quantization, roundoff error, channel estimation, fixed-point calculation
\end{abstract}

\section{INTRODUCTION}

Nowadays, multicarrier modulations are becoming moremore frequently adapted for the physical layer of digital data transmission systems. To avoid interferences between consecutive symbols, guard periods are applied to separate the symbols. These guard periods are such predefined blocks, which are concatenated to the transmitted data.

The Golay sequences are such special sequences with advantageous correlation properties [1] which can be used as guard intervals and for parameter estimation as well. They are applied in various standards such as IEEE 802.11ad and 3G CDMA. There are special complementary Golay sequences, which do not only separate the blocks from each other, but they can be used to estimate the parameters of the transmission such as the channel impulse response (CIR) [2], timing and frequency error [3].

To estimate the CIR from the received complimentary Golay sequences, a special lattice filter, a Golay correlator can be be applied [4]. It calculates the correlation of the incoming signal with the ideal Golay sequences, and the sum of these correlations will result in the CIR [2], [5]. The channel frequency response (CFR) can be also directly estimated based these sequences using the fast Fourier transform (FFT) [2], but it has higher complexity than the Golay correlator. The Golay correlator has a relatively low complexity: it requires only adders and multipliers with values -1 and 1 , as it can be seen in Fig. 1.

Besides these estimation method's complexity, their sensitivity to quantization effects has to be taken also into account Nevertheless the current analog-digital-converters (ADC) support only $8-12$ bits resolution within the $\mathrm{GHz}$ band, also the analog data are quantized to these bits. This means, that the digital signal differs from the analog, quantization errors may appear. This is a nonlinear error, which distorts the original

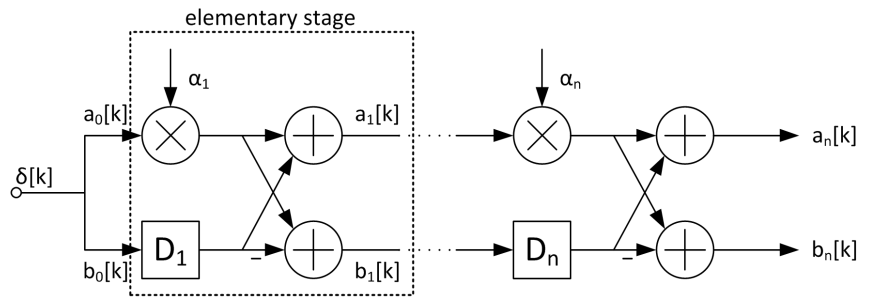

Fig. 1. Schematic structure of the Golay generator.

signal and the estimations as well. In this paper, we also investigate the effects of the quantization. The error caused by the limited resolution on the estimation of the CIR/CFR through the Golay correlator and through the FFT is compared.

The paper is organized as follows. In the next section, the theory for the generation of the complimentary Golay sequence and their application for channel estimation are discussed. Also the fixed-point arithmetical unit is shortly described which is used throughout the simulations. Section III presents the description and the results of the simulations. Finally, the paper is concluded in Section IV.

\section{THEORY}

\section{A. Golay Sequences}

1) Generation of Complimentary Golay Sequences: The Golay sequence pairs, $a[k]$ and $b[k]$ are such $N$ element long sequences with units $\{+1,-1\}$ for which the sum of their autocorrelation functions $\left(R_{A}[k], R_{B}[k]\right)$ is zero, except when the discrete time index $k$ is zero [1]

The following efficient algorithm can be used for generating the corresponding Golay sequences with a length of $N$, where $N$ is a power of 2 [4]:

$$
\begin{aligned}
a_{0}[k] & =b_{0}[k]=\delta[k], \\
a_{n}[k] & =\alpha_{n} \cdot a_{n-1}[k]+b_{n-1}\left[k-D_{n}\right], \\
b_{n}[k] & =\alpha_{n} \cdot a_{n-1}[k]-b_{n-1}\left[k-D_{n}\right],
\end{aligned}
$$

where $\delta[k]$ denotes the Kronecker delta function, $n$ is the index number of the stage in the generator $\left(n_{\max }=\log _{2} N\right)$, $\alpha_{n}$ contains the multiplication coefficients, and $D_{n}$ is the number of the delay elements (see Fig. 1 and Tab. I). 
TABLE I

APPLIED $\alpha_{n}$ AND $D_{n}$ VALUES

\begin{tabular}{|c|c|c|}
\hline$N$ & $\alpha_{n}$ & $D_{n}$ \\
\hline 16 & {$[+1,+1,+1,-1]$} & {$[8,1,2,4]$} \\
\hline 32 & {$[-1,+1,-1,+1,-1]$} & {$[1,4,8,2,16]$} \\
\hline 64 & {$[+1,+1,-1,-1,+1,-1]$} & {$[2,1,48,16,32]$} \\
\hline 128 & {$[-1,-1,-1,-1,+1,-1,-1]$} & {$[1,8,2,4,16,32,64]$} \\
\hline
\end{tabular}

TABLE II

APPLIED $\alpha_{n}^{\prime}$ AND $D_{n}$ VALUES FOR COMPLEX-VALUED SEQUENCES

\begin{tabular}{|c|c|c|}
\hline$N$ & $\alpha_{n}^{\prime}$ & $D_{n}$ \\
\hline 32 & {$[+\mathrm{j},+1,-1,-1,-1]$} & {$[8,1,2,4]$} \\
\hline 64 & {$[-1,-\mathrm{j},-1,-1,+1,-1]$} & {$[1,4,8,2,16]$} \\
\hline 128 & {$[+\mathrm{j},-1,+1,-1,+1,-1,-1]$} & {$[1,8,2,4,16,32,64]$} \\
\hline
\end{tabular}

2) Complex-valued Golay Vequences: These days, the complex-valued transmission methods - with IQ modulation - like e.g. OFDM, FBMC are widely applied in several standards. If these techniques are used for data encoding, then it is beneficial, that the applied Golay sequences are generated straightaway as complex valued signals. The complex valued Golay sequences can be generated directly with the extension of the generator with complex rotation [2].

In this paper, the lengths of the two complementary Golay sequences are $N=[32,64,128]$, which are denoted by $g_{A}$, $g_{B}$. Their inverted versions are $-g_{A},-g_{B}$. If the length of the sequences is also marked, then they are referred to as $g_{A, 32}$, $g_{A, 64}$ etc. The applied $\alpha_{n}^{\prime}$ and $D_{n}$ values for the generators for complex channels are given in Tab. II [2].

\section{B. Channel Estimation}

The multipath radio channel distorts the transmitted signal, so the received data have to be compensated by the inverse of the CIR. To perform this compensation, the CIR/CFR has to be estimated. Therefore predefined sequences are added to the message as a preamble, which are known by the receiver. The estimation of the CIR can be calculated based on the received and ideal, predefined sequences. In this paper, the applied Channel Estimation Field (CEF) for the contains complexvalued Golay sequences $-g_{U}$ and $g_{V}$ which are built up from $g_{A}$ and $g b$ sequences - as shown in Fig. 2 [2].

\begin{tabular}{|l|c|c|c|c|c|c|c|}
\hline$-g_{B}$ & $-g_{A}$ & $g_{B}$ & $-g_{A}$ & $-g_{B}$ & $g_{A}$ & $-g_{B}$ & $-g_{A}$ \\
\hline
\end{tabular}

Fig. 2. Structure of applied preamble to CIR-estimation.

For the simulations, a baseband-equivalent channel model is applied, where the transmitted signal $x[k]$ is filtered by the channel impulse response $h[k]$. The multipath channel is modeled as a FIR-filter. Furthermore, an Additive, White Gaussian Noise (AWGN) $w[k]$ is taken into account. The received signal $y[k]$ can be expressed as

$$
y[k]=x[k] * h[k]+w[k] .
$$

1) Golay Correlator Based Channel Estimation: In this paper, the complex valued Golay sequences $g_{A}$ and $g_{B}$ are applied. If the incoming CEF contains $g_{U}$ and $g_{V}$ sequences, then with the aid of their correlations with the ideal $g_{A}$ and $g_{B}$ the CIR can be perfectly estimated, as long as the channel noiseless.

If the transmitted signal $x[k]$ is the $g_{A}[k]$ sequence, then the received signal can be expressed as

$$
y[k]=g_{A}[k] * h[k],
$$

and the resulting correlated signal can be calculated as

$$
R_{A}[k]=g_{A}[k] * g_{A}[k] * h[k] .
$$

Similarly, if $x[k]$ is the $g_{B}[k]$ sequence, then the received and correlated signal can be given as

$$
R_{B}[k]=g_{B}[k] * g_{B}[k] * h[k] .
$$

Sum of the shifted versions of the correlations (4) and (5) results in the estimated CIR $\widetilde{h}[k]$, which can be expressed as

$$
\begin{aligned}
\widetilde{h}[k] & =R_{A}[k]+R_{B}[k], \\
\widetilde{h}[k] & =\left(g_{A}[k] * g_{A}[k]+g_{B}[k] * g_{B}[k]\right) * h[k] .
\end{aligned}
$$

As the $g_{A}$ and $g_{B}$ sequences are complementary to each other, therefore (7) can be simplified according to Sec. II-A1, which results in $\widetilde{h}[k]=h[k][2]$.

2) Fast Fourier Transform Based Channel Estimation: Instead of the time-domain approach, the CFR can calculated in the frequency domain with the aid of the FFT. Let $Y_{U}$ and $Y_{V}$ be the Fourier Transform of the incoming Golay sequences $y_{U}$ and $y_{V}$, furthermore $G_{U}$ and $G_{V}$ the previously calculated and stored spectral values of the transmitted $g_{U}$ and $g_{V}$ sequences. The CFR can be estimated separately using the Fourier transforms of the sequences as

$$
\begin{aligned}
\widetilde{H}_{U}[i] & =Y_{U}[i] / G_{U}[i], \\
\widetilde{H}_{V}[i] & =Y_{V}[i] / G_{V}[i] .
\end{aligned}
$$

The estimated CFR $(\widetilde{H}[i])$ is given by averaging the two estimates:

$$
\widetilde{H}[i]=\frac{\widetilde{H}_{U}[i]+\widetilde{H}_{V}[i]}{2} .
$$

If the CIR is needed, then it can be calculated by applying the inverse FFT to the CFR. It is important to note that some spectral values in $G_{U}$ and $G_{V}$ might be zero. To avoid the division by zero, the corresponding values have to be interpolated or substituted.

\section{Fixed-point Arithmetic Unit}

This unit is an custom-developed toolbox for MATLAB to simulate the operations of a fixed-point arithmetic unit and the effects of quantization. The numbers are stored and represented vectors with binary form. The length of the vector represents the Word Length (WL), where the last number of bits give the Fraction Length (FL); a further bit stores the sign of the number. This number representation is shown in Fig. 3. 


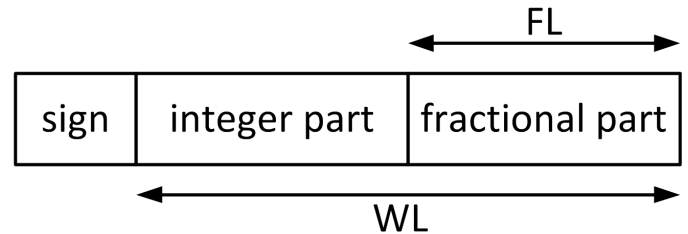

Fig. 3. Structure of the fixed-point numbers.

For the simulations, these parameters can be tuned. Besides setting the fixed point representations for the calculations, the type of rounding is also customizable, there are the following three options: "rounding towards zero", "rounding towards infinite" and "rounding to the nearest represented unit".

The functions have the following structure: the numbers have to be converted to this format through a quantizer function. The numbers are stored in a vectors format, but they can be converted back to normal form through the corresponding function. Currently the addition and the multiplication operations are supported. Besides these functions, some other functionality area also supported: multiplication by -1 , division by $2^{k}$ etc.

In this toolbox, the applied operations are not limited to the real numbers, the functions can handle complex valued numbers as well. Their real and imaginary parts are stored separately and the operations are executed according to rules of complex algebra.

\section{Simulations}

In this section, the results of the simulations are presented, two different scenarios are investigated where complex valued channel model is applied. Firstly, a COST 207 channel model [6] for typical urban area with 6 taps is applied without AWGN. During the second scenario, AWGN is also added to the signal to compare the effect of the channel and the quantization noise on the two estimation methods. For both scenarios, the transmitted signal consists only from a preamble according to the Fig. 2 because other elements are not required for channel estimation.

The Mean Square Error (MSE) of the quantized parameter estimation is calculated and used for the evaluation, where taps of the ideal channel are used as reference values. The estimated CIR is calculated by Golay correlator and by Fourier transform as well and their results are compared to the reference. During the simulations the sampling frequency is normalized to unity, furthermore, perfect synchronization is assumed.

\section{A. COST 207 Channel Model without Noise}

In this scenario, the Golay correlator contains $[5,6,7]$ stages, so the length of the sequences is $[32,64,128]$. During the simulations, the FL is set from 2 bits up to 32 bits in steps of 2 bits. For each sequence length and FL-value, 20 different channels are generated, and for each of them the MSE is calculated and averaged. The MSE results in function of the FL are presented in Fig. 4 and Fig. 5 for the sequence length

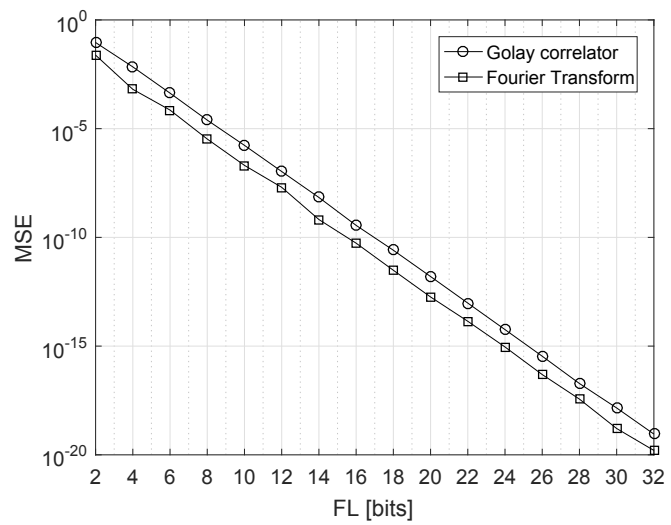

Fig. 4. MSE of quantized CIR-estimation for noiseless COST 207 channel model $(N=32)$.

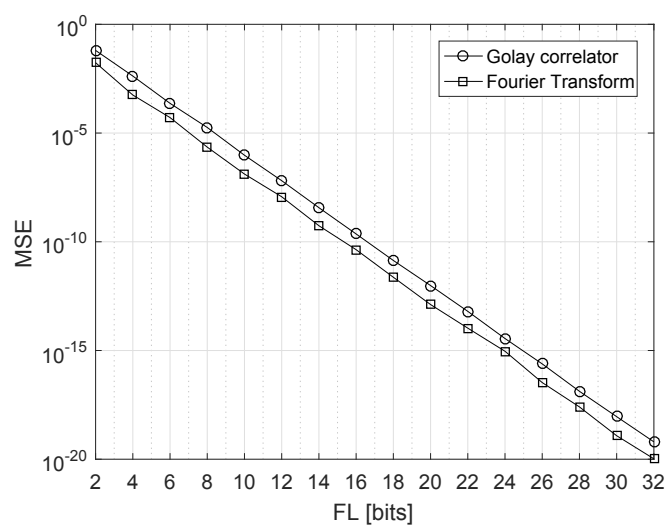

Fig. 5. MSE of quantized CIR-estimation for noiseless COST 207 channel model $(N=64)$.

of $N=32$ and $N=64$ respectively. The results show that the two curves are almost identical, the MSE is not strongly influence by the length of the sequence, but it can be stated that the channel estimation method using FFT performs slightly better than the Golay correlator based approach.

\section{B. COST 207 Channel Model with AWGN}

During the second scenario, the COST 207 channel model is used as well, but an AWGN is added to the signal, where the SNR is set up between the values 10,15 and $20 \mathrm{~dB}$. The applied Golay correlator is set up of 5 stages, so the applied sequence has $N=32$ elements. During the simulations, the FL is set from 2 bits up 32 bits in steps of 2 bits. At each sequence length and FL-value 20 different channels are generated and for each of them the MSE is calculated and averaged.

These MSE values are interpolated in form of $A \cdot F L^{B}+C$, because this interpolation gives a suitable curve fitting: the coefficient of determination $R^{2}$ are calculated as well. These values give a relegability of 0.96 or more (see Tab. III). These 

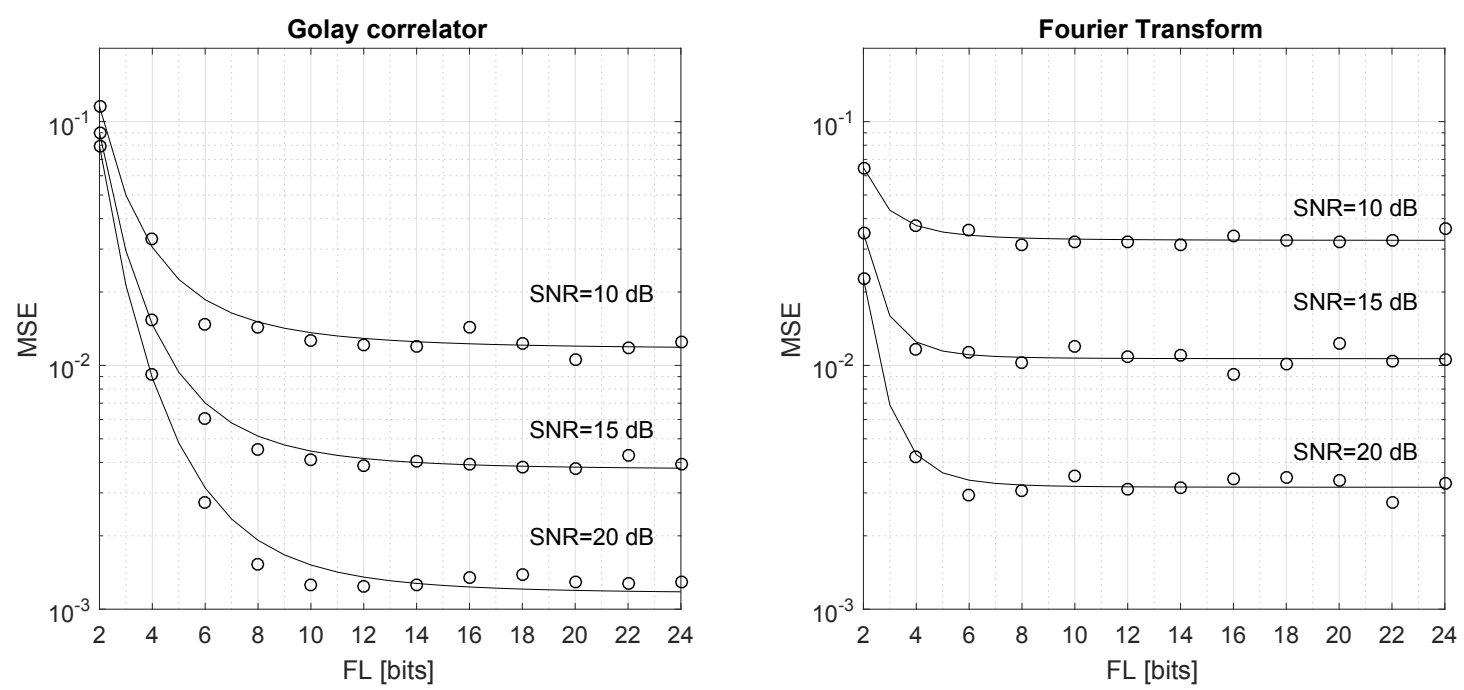

Fig. 6. MSE of the quantized CIR-estimation based on Golay correlator and FFT for COST 207 channel model with noise.

TABLE III

$R^{2}$ COEFFicients OF INTERPOLATIONS

\begin{tabular}{|c|c|c|}
\hline$R^{2}$ & Golay correlator & Fourier transform \\
\hline $10 \mathrm{~dB}$ & 0.9967 & 0.9684 \\
\hline $15 \mathrm{~dB}$ & 0.9996 & 0.9800 \\
\hline $20 \mathrm{~dB}$ & 0.9999 & 0.9966 \\
\hline
\end{tabular}

simulated and interpolated results are presented in Fig. 6 in function of the FL.

The results show, that there is only one dominant source of noise: if the applied resolution is very low, only a few bits, then the effect of the channel's noise is negligible and the quantization noise is more significant to the MSE. On the other hand if the resolution is improving then the effect of the quantization does not influence significantly the precision of the CIR estimation. In this case, the MSE of the CIR estimation is converging to an error floor, which can be determined, because it depends only on the SNR and not on the FL.

\section{CONCLUSION}

In this paper, the effects of the quantization for two channel estimation methods - the Golay correlator based and the FFT based - is investigated and presented. We have shown that in noiseless channels the FFT based approached has a slightly lower sensitivity to quantization errors. On the contrary if channel noise is introduced the Golay correlator outperforms the FFT based estimation method. Through simulations we stated that it is not necessary to increase the resolution of the ADC without limits, because the quantization noise does not influence the precision of the estimations after a certain FL value, SNR value is more dominant.

\section{ACKNOWLEDGMENT}

This work was supported by the János Bolyai Research Fellowship of the Hungarian Academy of Sciences.

\section{REFERENCES}

[1] M. J. E. Golay, "Complementary series," IRE Transactions on Information Theory, vol. 7, no. 2, pp. 82-87, Apr. 1961, DOI: 10.1109/TIT.1961.1057620.

[2] B. Csuka and Zs. Kollár, "Software and hardware solutions for channel estimation based on cyclic golay sequences," Radioengineering, vol. 4, no. 25, pp. 801-807, Dec. 2016, DOI: 10.13164/re.2016.0801.

[3] Z. Luo, J. Zheng, J. Zhu, and E. Zhang, "Golay complementary pair aided time synchronization method for OFDM systems," in 2012 IEEE 14th International Conference on Communication Technology, Nov 2012, pp. 166-170.

[4] P. G. Donato, M. A. Funes, M. N. Hadad, and D. O. Carrica, "Optimised Golay correlator," Electronics Letters, vol. 45, no. 7, pp. 380-381, March 2009, DOI: 10.1049/el.2009.2923.

[5] M. Lei and Y. Huang, "CFR and SNR Estimation Based on Complementary Golay Sequences for Single-Carrier Block Transmission in $60-\mathrm{GHz}$ WPAN," in IEEE Wireless Communications and Networking Conference, 2009, Apr. 2009, pp. 1-5, DOI: 10.1109/WCNC.2009.4917623.

[6] "COST 207 - digital land mobile radio communications (Final reports)," Office for Official Publications of the European Communities, Luxembourg, 1989. 